\title{
The Role of Selenium in Sepsis
}

\author{
Jennifer McArthur* and Michael W. Quasney
}

Division of Pediatric Critical Care, Department of Pediatrics, Medical College of Wisconsin, and the Children's Research Institute, Children's Hospital of Wisconsin, Milwaukee, WI

\begin{abstract}
Selenium is a key component of many important enzyme systems, including the glutathione peroxidase (GPX) family of enzymes. Glutathione peroxidases are primarily involved in repairing cell membranes damaged by lipid peroxidation caused by reactive oxygen species. The glutathione peroxidases may also have an anti-inflammatory role. Sepsis is known to cause a significant degree of oxidative stress. The selenium containing glutathione peroxidases play an important role in neutralizing the oxidative damage from sepsis. Selenium supplementation to patients with sepsis shows a potential to improve important outcomes such as mortality and organ dysfunction. However, larger more definitive trials are needed to define the optimal dosing and timing of supplementation.
\end{abstract}

Keywords: Selenium, sepsis, oxidative stress, reactive oxygen species, glutathione peroxidase.

\section{OXIDATIVE STRESS IN SEPSIS}

Reactive oxygen species (ROS) have many diverse roles in biological systems including the stimulation of cell proliferation, maintenance of vascular tone, and migration and signaling of inflammatory cells to sites of inflammation. Under normal conditions of health, there is a delicate balance between the ROS, and the body's scavenging systems for these potentially harmful mediators. However, significant oxidative stress occurs during sepsis and septic shock when there is activation of granulocytes and endothelial cells resulting in an oxidative burst and overabundance of ROS. The body's endogenous antioxidant mechanisms and ROS scavenging systems are overwhelmed resulting in protein oxidation, lipid peroxidation leading to cellular membrane damage, and DNA damage all of which result in irreversible cellular damage and contribute to the multiple organ dysfunction observed in sepsis.

The oxidative stress observed in sepsis is evident by several studies. Total plasma antioxidant capacity (TAC) is a measure of the cumulative ability of a patient's antioxidant systems to scavenge free radicals. Investigators have demonstrated that while TAC between septic patients and healthy volunteers are similar, TAC levels decline over time in the septic patients suggesting a loss in the ability of septic patients to scavenge the ROS. In vitro studies in which human umbilical vein endothelial cells are treated with plasma from patients with septic shock have demonstrated that high amounts of ROS are produced [1] and intracellular glutathione stores, the most abundant antioxidant, are depleted [2]. The amount of ROS produced correlated to the severity of illness and pretreatment with n-acetylcysteine or glutathione decreased ROS production and cell death [2].

*Address correspondence to this author at the Division of Pediatric Critical Care, Department of Pediatrics, Medical College of Wisconsin, and the Children's Research Institute, 9000 W. Wisconsin Ave, MS681, Milwaukee, WI 53201, USA; Tel: (414) 266-3360; Fax: (414) 266-3563;

E-mail: jmcarthur@mcw.edu
Diminished glutathione levels have also been demonstrated in muscle biopsies from adult patients with sepsis compared with non-septic controls undergoing hip surgery and that the degree of mitochondrial dysfunction was highly correlated with glutathione levels and the patient's outcome [3]. Diminished glutathione synthesis and subsequently whole blood glutathione levels have also been observed in septic pediatric patients [4] .

Serum levels of other antioxidants have also been demonstrated to be decreased in patients with sepsis. For example, adult septic patients have significantly lower concentrations of the antioxidants Vitamin A, Vitamin E, beta carotene and lycopene compared to healthy controls [5]. In addition, higher levels of thiobarbituric acid-reactive substances thought to represent increased lipid peroxidation due to oxidative stress were demonstrated in the septic group of patients. Increased xanthine oxidase activity has also been observed in adult patients with sepsis syndrome and multiple organ dysfunction compared with non-septic ICU patients and healthy controls. Xanthine oxidase is an enzyme activated during ischemia reperfusion injury, a process during which ROS are formed. The patients with sepsis syndrome also had elevation of other serum markers of oxidative stress such as malondialdehyde and lipid peroxidation lending further support that sepsis induced oxidative stress [6]. Plasma protein carbonyl concentrations, an indicator of protein oxidation, have also been observed to be elevated in both patients with sepsis syndrome as well as trauma patients compared with healthy controls. Protein carbonyl concentrations in bronchoalveolar (BAL) fluid were also high in the critically ill patients approaching the levels seen in plasma. On the other hand, levels of thiobarbituric acid-reactive substances, indicators of lipid peroxidation, were not significantly different between septic patients, trauma patients or healthy controls [7]. Thus, reactive oxygen species produced during critical illness result in oxidative damage to proteins both globally, as 
indicated by elevated levels in plasma, as well as within the lung, as indicated by elevated levels in BAL fluid.

\section{SELENIUM , SELENOPROTEINS, AND SEPSIS}

The body has extensive defense mechanisms against the ROS produced during sepsis and other critical illnesses. One of these mechanisms involves a group of proteins that utilize selenium, a trace element that is essential for the formation of the amino acid selenocysteine. Selenocysteine is a key component of a number of selenoproteins. Many enzymes containing selenium have selenocysteine at their active site and play important roles in maintaining an appropriate oxidative balance. The first selenoenzymes to be described belong to the glutathione peroxidase (GPX) family, (glutathione peroxidases 1 through 4). This family of selenoenzymes plays an important role as antioxidants as they convert hydrogen peroxide to water by utilizing glutathione. Glutathione peroxidases are primarily involved in repairing cell membranes damaged by lipid peroxidation caused by reactive oxygen species. The glutathione peroxidases may also have an anti-inflammatory role (Fig. 1.). Selenium supplementation has been shown in cell culture models to down regulate $\mathrm{NF}-\kappa \beta$ transcription, a crucial mediator in inflammatory pathways. It is postulated that the mechanism by which selenium down regulates NF$\kappa \beta$ transcription is through the action of the glutathione peroxidases [8].

Other selenoenzymes include the family of thioredoxin reductases and iodothryinone deiododinases. The thioredoxin reductases also play an important antioxidant role by reducing thioredoxin and are involved in the recycling of dehydroascorbate to ascorbate (Vitamin C) as well as ubiquinol to ubiquinone (Coenzyme Q-10). The iodothyrinone deiododinases are important in the synthesis of thyroid hormones [9]. In a trial of selenium supplementation in 41 adults with severe sepsis, the patients who were randomized to receive selenium had earlier normalization of their thyroid hormone parameters. However, the authors did not feel this was a direct effect from the selenium supplementation. Rather, they felt the patients were clinically improving and therefore, their clinical improvement was the cause of their thyroid parameters [10]. Thus, selenium containing enzymes play a central role in the host's strategies to counter the oxidative stresses of sepsis.
There are also a number of non-enzymatic selenoproteins that are involved in regulating selenium levels in the body. For example, the serum protein selenoprotein $\mathrm{P}$ plays an important role in the distribution of selenium in various tissues in the body and is a good measure of selenium nutritional status. There is also evidence that selenoprotein $\mathrm{P}$ may play a role in protecting endothelial cells from oxidative damage [11]. When selenium deficient rats are given low doses of diquat, the animals die within hours of liver necrosis. However, when the rats are given selenium prior to diquat injection, liver necrosis does not occur. Selenoprotein $\mathrm{P}$ is detected in the plasma shortly after the selenium injection and is believed to play an important role in protecting liver endothelial cells.

If selenium plays an important role in the body's defense mechanisms against oxidative stress, and sepsis has been shown to result in an oxidative stress, is selenium homeostasis altered during sepsis? Several studies appear to support decreased selenium during sepsis. Serial plasma selenium concentrations appear to be decreased in critically ill patients overall; 134 patients admitted to an adult medical and surgical ICU demonstrated decreased serum levels of selenium compared to healthy controls [12]. A significant negative correlation was found between plasma selenium levels and severity of illness scores, with the most severely ill patients having the lowest selenium levels. Low selenium levels also appeared to be associated with an increased risk of nosocomial infections, organ failure and mortality. Furthermore, patients with sepsis, SIRS and evidence of hypoperfusion had the lowest selenium levels [12]. Similar results were observed in adults with varying degrees of SIRS, sepsis and septic shock [13]. Serum selenium levels were low in all groups of patients compared to controls, and the greatest decrease over time was seen in the patients with organ failure, particularly in those with infection.

\section{ANTI-OXIDANT THERAPY IN SEPSIS}

\section{N-Acetylcysteine}

With the accumulating evidence that ROS are elevated in patients with sepsis, treatment or dietary supplementation with anti-oxidants to act as oxygen free radical scavengers might limit the damage caused by ROS. Indeed, several studies have demonstrated a benefit of anti-oxidants in critically ill patients. For example, supplementation with the

Selenium in Glutathione Peroxidase System

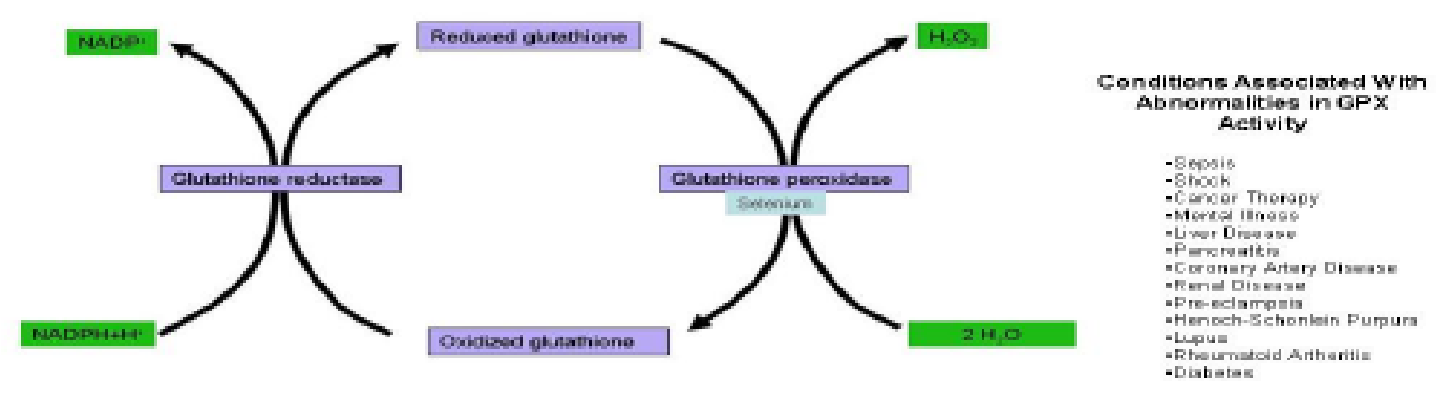

Fig. (1). 
glutathione precursor n-acetylcysteine, the most ubiquitous antioxidant in the human body and a substrate for the glutathione peroxidase system, has been investigated in patients with sepsis and lung injury. In a double blinded, placebo controlled study of 22 septic patients, subjects were randomized within 4 hours of diagnosis to receive nacetylcysteine) or placebo [14]. The most significant improvements were noted in pulmonary function as the group treated with $\mathrm{N}$-acetylcysteine had improved $\mathrm{PaO} 2 / \mathrm{FiO} 2$ ratios and static lung compliance at 24 hours after infusion. In survivors, those who received nacetylcysteine had significantly shorter ventilator days $(7.2 \pm 2$ days vs $20 \pm 7$ days, $\mathrm{p}<0.05)$. No differences were observed between the n-acetylcysteine-treated and placebo groups in several hemodynamic parameters measured by pulmonary artery catheters or in plasma concentrations of cytokines including TNF- $\alpha$, IL-6, IL-8, IL-10, or soluble TNF- $\alpha$ receptor-p55 [14]. In contrast, cardiac indices as well as hepatic blood flow and microsomal hepatic function, as measured by the ability to metabolize lignocaine, were improved in another randomized study of 60 adults with septic shock who received either n-acetylcysteine or placebo [15]. NF- $\kappa \beta$ activation and IL-8 serum levels were lower while IL-6 and soluble ICAM-1 serum levels were unchanged in a study of 20 adults with sepsis [16]. This provides some insight into the possible mechanism by which $n$-acetylcysteine may have an anti-inflammatory influence as $\mathrm{NF}-\kappa \beta$ is an important initial step in the induction of the inflammatory cascade.

Clinical trials have also been performed utilizing $\mathrm{N}$ acetylcysteine along with other anti-oxidant cocktails to explore potential synergistic effects. For example, Ortolani et al. randomized 30 patients with septic shock to receive placebo, glutathione or glutathione with n-acetylcystein [17]. Lipid peroxidation was measured by both exhaled ethane and plasma malondialdehyde levels and cell membrane phospholipid damage was measured by erythrocyte membrane deformability. Improvement was observed in all markers of oxidative stress in both treatment arms, with the most improvement seen in the group receiving both glutathione and n-acetylcysteine [17]. Hemodynamic parameters such as heart rate, cardiac index, and systemic vascular resistance were improved in a cohort of adults with sepsis who received a combination antioxidant therapy of nacetylcysteine, ascorbic acid and $\alpha$-tocopherol [18]. Basal vitamin $\mathrm{C}$ levels were low and evidence of oxidative stress such as total lipid peroxides, malondialdehyde, and redox reactive iron, were elevated in all patients in the study. Neither patients receiving the antioxidant therapy or placebo demonstrated an improvement in these measures during the 6 hour time course of the infusion. It is possible that no improvement was seen in serum markers of oxidative stress because they were drawn too early. In the study by Ortolani et al. patients were also given n-acetylcysteine (+/glutathione) but markers of oxidative stress were not evaluated until 3 and 5 days post infusion.

\section{Anti-oxidant Treatment Regimens Containing Selenium}

In the past decade, several trials in critically ill patients have investigated the use of antioxidant cocktails which include selenium. One of the first of these trials involved 326 adult patients admitted to an ICU with systemic inflammatory response syndrome (SIRS). The underlying etiology for the patient's SIRS included trauma, surgery or sepsis. Patients were randomized to standard enteral nutrition (Osmolite $\mathrm{HN}$ ) or the study formula which contained increased levels of omega-3 fatty acids, arginine, betacarotene, vitamin $\mathrm{E}$ and selenium. While there was no difference in overall mortality between the 2 study groups, subgroup analysis demonstrated that in those patients who tolerated at least $821 \mathrm{~mL} /$ day of either standard or study formula, patients who received study formula had a decreased length of hospital stay. The greatest benefit appeared to be in the subgroup of patients with sepsis who demonstrated not only a decreased length of hospital stay but also a significant reduction in nosocomial infections [19]. A second large trial utilizing selenium in the treatment regimen involved 165 patients requiring mechanical ventilation for sepsis or septic shock [20]. Patients were randomized to receive enteral nutrition with a standard formula or a study formula that contained higher levels of omega-3 fatty acids and $\gamma$-linolenic oil, less omega- 6 fatty acids, and higher doses of the antioxidant vitamins $\mathrm{E}$ and $\mathrm{C}$ and selenium. Patients receiving the study formula had an improvement in their mortality rate, oxygenation, ventilator-free days, ICUfree days, and less development of new organ dysfunction. A third randomized trial of 55 adults with sepsis evaluated a study feeding regiment that included increased amounts of glutamine, butyrate, beta-carotene, vitamins $\mathrm{E}$ and $\mathrm{C}$, zinc and selenium. While there was no improvement observed in mortality, hospital length of stay or ICU stay, those patients receiving the study feeding regimen demonstrated significantly faster improvement of organ dysfunction. Levels of vitamins $\mathrm{C}$ and $\mathrm{E}$, beta-carotene, glycine, ornithine, serine and arginine were all increased in the study group as compared to the control group demonstrating an ability of these critically ill patients to absorb specific enteral supplements [21].

Clinical trials of antioxidant cocktails have also been performed in critically ill trauma, burn, and post-operative surgical patients. In one trial, 41 severely burned patients were randomized to receive placebo or supplementation with copper, selenium, and zinc. Patients receiving supplementation significantly improved plasma and skin concentrations of selenium, zinc and glutathione peroxidase and had a significant reduction in nosocomial infections when compared to placebo [22]. A similar trial included 200 adult patients admitted to the ICU with evidence of organ failure after major trauma, cardiac surgery or subarachnoid hemorrhage. Patients were randomized to receive 5 days of intravenous supplementation with vitamin $C$, vitamin $B_{1}$, selenium and zinc or placebo. Patients receiving the study regimen demonstrated significantly higher levels of selenium, zinc and glutathione peroxidase compared to the control group. While levels of the inflammatory marker Creactive protein (CRP) were decreased significantly more in the group receiving the study regimen when compared to the control group, there were no differences seen in infectious complications. Furthermore, overall hospital length of stay was not different between the two groups. However, in the subgroup of surviving trauma patients, those in the antioxidant supplementation group demonstrated a shorter length of hospital stay [23]. Recently a large, retrospective review was reported of adults with trauma before and after 
the initiation of protocolized administration of an antioxidant cocktail containing ascorbic acid, $\alpha$-tocopherol and selenium to all acutely injured patients [24]. Patients who were admitted after the implementation of the antioxidant protocol had improved outcomes including hospital mortality, length of ICU stay and length of hospital stay, with the most significant improvements noted in the sickest patients with a probability of survival $<50 \%[24]$.

Thus, sizable clinical trials in critically ill adults have been performed that appear to demonstrate a clinically meaningful improvement in those patients receiving supplementation with antioxidant regimens that contain selenium. However, it may be impossible to ascertain the role of selenium in the outcome of these trials or the benefit of selenium itself because of the variations seen in the treatment regimens.

A few studies exist in both animal models of sepsis as well as human studies, however, in which the primary treatment regimen was selenium. For example, in a sheep model of sepsis, animals treated with a bolus of selenium 9 hours after induction of peritonitis had improved hemodynamic parameters and prolonged survival time compared to animals given a continuous infusion of selenium or placebo [25]. Human trials are also beginning to demonstrate improvement in a variety of outcomes. A randomized placebo controlled trial of selenium supplementation in 42 adult patients with SIRS demonstrated significantly higher selenium levels and glutathione peroxidase activity after 3 days of supplementation when compared to the placebo group [26]. While there was no difference noted in the overall mortality between the two groups, the severity of illness scores improved significantly in the selenium treated group. Recently, a larger randomized, placebo controlled trial of selenium supplementation in adults with SIRS, sepsis and septic shock demonstrated a reduced 28 -day mortality rate $(42.4 \%$ vs $56.7 \%$ in the placebo group; $p=.049$ ) [27]. Patients were randomized to receive placebo or selenium as an initial bolus of $1000 \mu \mathrm{g}$ over 30 minutes followed by 14 daily continuous infusions of $1000 \mu \mathrm{g} / \mathrm{day}$. Patients in the selenium-supplemented group had selenium levels and glutathione peroxidase-3 activity in the upper normal range throughout the treatment time while patients in the placebo group had significantly lower levels throughout the course of the study. The largest difference in the 28-day mortality was seen in the most critically ill patients: those with septic shock and disseminated intravascular coagulation, patients with APACHE III scores $\geq 102$, or patients with $>3$ organ dysfunction [27]. However, not all clinical trials utilizing selenium in critically ill patients have demonstrated a benefit. Sixty adults with severe septic shock were randomized to receive placebo or selenium supplementation of $4000 \mu \mathrm{g}$ by continuous infusion on the first day followed by $1000 \mu \mathrm{g} /$ day by continuous infusion for the subsequent 9 days [28]. No difference was found between the two groups in mortality, hospital length of stay, rates of organ failure or nosocomial infections. The lack of benefit in the seleniumtreated group may have been due to a less than optimal dosing schedule, and this protocol did not use an initial bolus dose as many other trials showing benefit utilized. It has been suggested that the initial bolus may down regulate NF- $\kappa \beta$ resulting in decreased production of inflammatory cytokines.

\section{CONCLUSION}

Oxidative stress during sepsis results in a number of reactive oxygen species that augment the molecular and cellular damage that occurs and can lead to multiorgan system failure. The host defense mechanisms against these oxidative stresses appear to be attenuated during sepsis. Supplementation of antioxidants, particularly selenium, is a potentially promising adjunctive therapy for patients with SIRS, sepsis and septic shock. It may play an important role both as an antioxidant through the glutathione peroxidase system and as an inhibitor of NF- $\kappa \beta$. Optimal dosing has yet to be determined in adults and no clinical trials in children with sepsis have yet to be performed. Further studies, particularly in children, are necessary to determine whether these early promising results utilizing selenium and other antioxidants prove to be beneficial in large randomized, placebo controlled trials.

\section{REFERENCES}

[1] Huet O, Obata R, Aubron C, et al. Plasma-induced endothelial oxidative stress is related to the severity of septic shock. Crit Care Med 2007; 35(3): 821-6.

[2] Huet O, Cherreau C, Nicco C, et al. Pivotal role of glutathione depletion in plasma-induced endothelial oxidative stress during sepsis. Crit Care Med 2008; 36(8): 2328-34.

[3] Brealey D, Brand M, Hargreaves I, et al. Association between mitochondrial dysfunction and severity and outcome of septic shock. Lancet 2002; 360(9328): 219-23.

[4] Lyons J, Rauh-Pfeiffer A, Ming-Yu Y, et al. Cysteine metabolism and whole blood glutathione synthesis in septic pediatric patients. Crit Care Med 2001; 29(4): 870-7.

[5] Goode HF, Cowley HC, Walker BE, Howdle PD, Webster NR. Decreased antioxidant status and increased lipid peroxidation in patients with septic shock and secondary organ dysfunction. Crit Care Med 1995; 23(4): 646-51.

[6] Galley HF, Davies MJ, Webster NR. Xanthine oxidase activity and free radical generation in patients with sepsis syndrome. Crit Care Med 1996; 24(10): 1649-53.

[7] Winterbourn CC, Buss IH, Chan TP, Plank LD, Clark MA, Windsor JA. Protein carbonyl measurements show evidence of early oxidative stress in critically ill patients. Crit Care Med 2000; 28(1): 143-9.

[8] Kretz-Remy C, Arrigo AP. Selenium: a key element that controls NF-kappa B activation and I kappa B alpha half life. Biofactors 2001; 14(1-4): 117-25.

[9] Kohrle J. The selenoenzyme family of deiodinase isozymes controls local thyroid hormone availability. Rev Endocr Metab Disord 2000; 1(1-2): 49-58.

[10] Angstwurm MW, Schopohl J, Gaertner R. Selenium substitution has no direct effect on thyroid hormone metabolism in critically ill patients. Eur J Endocrinol 2004; 151(1): 47-54.

[11] Burk RF, Hill KE, Motley AK. Selenoprotein metabolism and function: evidence for more than one function for selenoprotein P. J Nutr 2003; 133(5 Suppl 1): 1517S-20S.

[12] Forceville X, Vitoux D, Gauzit R, Combes A, Lahilaire P, Chappuis P. Selenium, systemic immune response syndrome, sepsis, and outcome in critically ill patients. Crit Care Med 1998; 26(9): 1536-44.

[13] Sakr Y, Reinhart K, Bloos F, et al. Time course and relationship between plasma selenium concentrations, systemic inflammatory response, sepsis, and multiorgan failure. Br J Anaesth 2007; 98(6): 775-84.

[14] Spapen H, Zhang H, Demanet C, Vleminckx W, Vincent JL, Huyghens L. Does N-acetyl-L-cysteine influence cytokine response during early human septic shock? Chest 1998; 113(6): 1616-24.

[15] Rank N, Michel C, Haertel C, et al. N-acetylcysteine increases liver blood flow and improves liver function in septic shock patients: 
results of a prospective, randomized, double-blind study. Crit Care Med 2000; 28(12): 3799-807.

[16] Paterson RL, Galley HF, Webster NR. The effect of Nacetylcysteine on nuclear factor-kappa B activation, interleukin-6, interleukin-8, and intercellular adhesion molecule-1 expression in patients with sepsis. Crit Care Med 2003; 31(11): 2574-8.

[17] Ortolani O, Conti A, De Gaudio AR, Moraldi E, Cantini Q, Novelli G. The effect of glutathione and N-acetylcysteine on lipoperoxidative damage in patients with early septic shock. Am J Respir Crit Care Med 2000; 161(6): 1907-11.

[18] Galley HF, Howdle PD, Walker BE, Webster NR. The effects of intravenous antioxidants in patients with septic shock. Free Radic Biol Med 1997; 23(5): 768-74.

[19] Bower RH, Cerra FB, Bershadsky B, et al. Early enteral administration of a formula (Impact) supplemented with arginine, nucleotides, and fish oil in intensive care unit patients: results of a multicenter, prospective, randomized, clinical trial. Crit Care Med 1995; 23(3): 436-49.

[20] Pontes-Arruda A, Aragao AM, Albuquerque JD. Effects of enteral feeding with eicosapentaenoic acid, gamma-linolenic acid, and antioxidants in mechanically ventilated patients with severe sepsis and septic shock. Crit Care Med 2006; 34(9): 2325-33.

[21] Beale RJ, Sherry T, Lei K, et al. Early enteral supplementation with key pharmaconutrients improves Sequential Organ Failure Assessment score in critically ill patients with sepsis: outcome of a randomized, controlled, double-blind trial. Crit Care Med 2008; 36(1): 131-44
[22] Berger MM, Eggimann P, Heyland DK, et al. Reduction of nosocomial pneumonia after major burns by trace element supplementation: aggregation of two randomised trials. Crit Care 2006; 10(6): R153.

[23] Berger MM, Soguel L, Shenkin A, et al. Influence of early antioxidant supplements on clinical evolution and organ function in critically ill cardiac surgery, major trauma, and subarachnoid hemorrhage patients. Crit Care 2008; 12(4): R101.

[24] Collier BR, Giladi A, Dossett LA, Dyer L, Fleming SB, Cotton BA. Impact of high-dose antioxidants on outcomes in acutely injured patients. JPEN J Parenter Enteral Nutr 2008; 32(4): 384-8.

[25] Wang Z, Forceville X, Van Antwerpen P, et al. A large bolus, but not a continuous infusion, of sodium selenite improves outcome in peritonitis. Shock 2009; 32(2): 140-6.

[26] Angstwurm MW, Schottdorf J, Schopohl J, Gaertner R. Selenium replacement in patients with severe systemic inflammatory response syndrome improves clinical outcome. Crit Care Med 1999; 27(9): 1807-13.

[27] Angstwurm MW, Engelmann L, Zimmermann T, et al. Selenium in Intensive Care (SIC): results of a prospective randomized, placebocontrolled, multiple-center study in patients with severe systemic inflammatory response syndrome, sepsis, and septic shock. Crit Care Med 2007; 35(1): 118-26.

[28] Forceville X, Laviolle B, Annane D, et al. Effects of high doses of selenium, as sodium selenite, in septic shock: a placebo-controlled, randomized, double-blind, phase II study. Crit Care 2007; 11(4): R73.

(C) McArthur and Quasney; Licensee Bentham Open.

This is an open access article licensed under the terms of the Creative Commons Attribution Non-Commercial License (http: //creativecommons.org/licenses/by-nc/3.0/) which permits unrestricted, non-commercial use, distribution and reproduction in any medium, provided the work is properly cited. 